\title{
Application of NOMA for cellular-connected UAVs: opportunities and challenges
}

\author{
Wee Kiat NEW ${ }^{*}$, Chee Yen LEOW ${ }^{1}$, Keivan NAVAIE ${ }^{2}$, \\ Yanshi $\mathrm{SUN}^{3} \&$ Zhiguo DING ${ }^{*}$ \\ ${ }^{1}$ Wireless Communication Centre, School of Electrical Engineering, Faculty of Engineering, \\ Universiti Teknologi Malaysia, Skudai 81310, Malaysia; \\ ${ }^{2}$ School of Computing and Communications, Lancaster University, Lancaster LA1 4WA, United Kingdom; \\ ${ }^{3}$ Key Laboratory of Wireless-Optical Communications, Chinese Academy of Sciences, \\ School of Information Science and Technology, University of Science and Technology of China, Hefei 230026, China; \\ ${ }^{4}$ School of Electrical and Electronic Engineering, The University of Manchester, Manchester M13 9PL, United Kingdom \\ Received 19 March 2020/Revised 20 June 2020/Accepted 17 July 2020/Published online 1 March 2021
}

\begin{abstract}
Unmanned aerial vehicles (UAVs) have gained considerable interests in numerous civil applications. To push forward its potentials, cellular-connected UAVs have been introduced. Nevertheless, cellular networks face several bottlenecks such as spectrum scarcity and limited concurrent connectivity. To address these issues, non-orthogonal multiple access (NOMA) can be adopted. NOMA provides several opportunities for cellular-connected UAVs such as larger rate region, balanced performance between system throughput and fairness, and reduced delay. In this paper, we review important findings of the related studies, and outline new opportunities and challenges in NOMA for cellular-connected UAVs. Monte-Carlo simulations are then performed to analyze the new aerial user's (AU)'s signal characteristics and evaluate the NOMA performance for co-existence of AU and terrestrial user (TU). Our preliminary results show that NOMA is a promising strategy for cellular-connected UAVs.
\end{abstract}

Keywords cellular-connected UAVs, non-orthogonal multiple access, signal characteristics, aerial user rate, terrestrial user rate

Citation New W K, Leow C Y, Navaie K, et al. Application of NOMA for cellular-connected UAVs: opportunities and challenges. Sci China Inf Sci, 2021, 64(4): 140302, https://doi.org/10.1007/s11432-020-2986-8

\section{Introduction}

Unmanned aerial vehicles (UAVs) have gained huge attentions in civil applications such as agriculture, construction, delivery, and surveillance [1]. Nevertheless, most of the commercial UAVs are restricted to visual line-of-sight (LOS) operations, where direct point-to-point links over unlicensed spectrum are used. To enable beyond visual LOS operations, researchers have recently introduced the concept of cellularconnected UAVs $[2,3]$. As the name suggested, the key idea of cellular-connected UAVs is to connect the UAVs to the cellular networks as aerial users (AUs). Given the wide availability of cellular infrastructure, the end users or terrestrial pilots can then remotely control the AUs over a wider geographical area. This opens up the potentials of UAV-based applications and innovates more UAV-related services.

To support new AUs, existing studies are relying on orthogonal multiple access (OMA) such as frequency division multiple access (FDMA), time division multiple access (TDMA), code division multiple access (CDMA), and orthogonal frequency division multiple access (OFDMA) [4,5]. In OMA, users are served using orthogonal resource blocks (RBs). This strategy helps to prevent multi-user interference in single-cell network. However, due to finite amount of RBs, the spectral efficiency and number of concurrent connectivity in OMA are limited. Concretely, if a user has a weak channel, its rate is likely to be low as defined by Shannon's capacity. In contrast, if the user has a strong channel but limited information to

\footnotetext{
* Corresponding author (email: aven.wknew@yahoo.com, zhiguo.ding@manchester.ac.uk)
} 
be transmitted, its rate might still be below its capacity. In both cases, orthogonal RB has to be assigned to the user and the same RB cannot be utilized by other users.

To overcome these limitations, non-orthogonal multiple access (NOMA) has been proposed for future wireless networks $[6,7]$. The key idea of NOMA is to leverage superposition coding (SC) at the transmitter and successive interference cancellation (SIC) at the receiver. This technique enables orthogonal RBs to be shared among multiple users. In this article, we focus on power domain NOMA. Power domain NOMA can be applied to both the uplink and the downlink. In the downlink, the base station (BS) superimposes and transmits user signals over the same orthogonal RB with distinctive power allocations. Upon receiving the signal, a weak user treats the co-channel interference as noise to decode its own signal, and a strong user performs SIC to decode its own signal. In the uplink, users transmit their own signals over the same orthogonal RB to the BS. The BS then performs SIC to decode the signal of each user. Therefore, using NOMA, the spectral efficiency and number of concurrent connectivity can be improved.

Unlike the terrestrial systems, additional motivations of applying NOMA to cellular-connected UAVs are as follows.

(1) Recent studies have shown that AUs experience a different propagation condition as compared to terrestrial users (TUs). Due to their high altitudes, AUs have higher probabilities of experiencing stronger communication links (e.g., LOS links) to the BSs than that of the TUs. Due to highly distinctive channels among users, NOMA provides a larger rate region [8]. Furthermore, as AUs could move in 3D space, this new feature can be exploited to improve the overall system performance.

(2) In downlink, the asymmetricity between AUs and TUs can be exploited [9]. Specifically, AUs experience a different propagation from TUs. Besides, TUs require a higher data rate for multimedia applications [10], whereas AUs typically require a lower data rate with high reliability for command and control (C\&C) links [11]. Such asymmetric characteristics enable NOMA to efficiently serve the TUs' links as usual while providing reliable connectivity to the AUs.

(3) In uplink, a larger macro-diversity can be obtained at AUs than that of TUs. This large macrodiversity provides higher degrees of freedom to the system, and hence one may further exploit this new feature to improve the overall performance. For instance, unlike TUs, AUs may use multiple BSs to perform cooperative SIC [12], or employ precoding [13] to improve their throughput, delay or fairness.

Nevertheless, the co-existence of AUs and TUs introduces new challenges to the cellular networks. The challenges mainly rise from two sides.

(1) Existing cellular infrastructures are designed to serve the TUs, where terrestrial BSs tilt their antennas downward. This enhances the desired signal and mitigates the intercell interference (ICI) of the TUs. Nevertheless, such tilting causes AUs to be served by the sidelobe of the BSs, and consequently they suffer a significant antenna gain reduction. Such tilting also generates coverage holes in the sky, leading AUs to be out of coverage at times [14]. Hence, the AUs' links are greatly compromised.

(2) AUs have different behaviors and characteristics as compared to the TUs. Due to 3D mobility, AUs have more versatile movements than that of TUs such as vehicles moving on the road. Such 3D mobility causes non-stationary time-varying channel, dynamic topologies, and frequent handover. Besides, in downlink, AUs suffer ICI from more neighboring BSs, whereas in uplink, AUs generate severe ICI to the TUs. In addition, AUs usually have asymmetric uplink and downlink rate demands, whereas TUs have more symmetric uplink and downlink rate demands. Therefore, significant variations in terms of performance and requirements between AUs and TUs are anticipated.

The main objective of this paper is to discuss the application of NOMA for cellular-connected UAVs, while emphasizing on the corresponding opportunities and challenges. To this end, we review important findings of the related studies, and outline new research opportunities and challenges in NOMA for cellular-connected UAVs. We then consider downlink NOMA for cellular-connected UAVs, where both AU and TU are present. Specifically, Monte-Carlo simulations are performed to analyze the new AUs' signal characteristics and to evaluate the performance of downlink NOMA for co-existing AU and TU. By investigating the impact of the network parameters, our results provide quantitative insights and reflect on the corresponding opportunities and challenges. Based on these preliminary results, we conclude the potentials of NOMA for cellular-connected UAVs.

The rest of the paper is organized as follows: Section 2 reviews the related work, and Section 3 outlines the opportunities and challenges. The simulation framework and performance metrics are detailed in Section 4, followed by the result discussion in Section 5. We then draw the conclusion in Section 6. 


\section{Related work}

Ground-to-air (G2A) channel experiences a different propagation from the ground-to-ground (G2G) channel [15-17]. With higher altitudes, AUs typically establish LOS links with their associated terrestrial BSs. The LOS links experience less obstacles in the air and weak reflections from the ground, and therefore the received signals are stronger. The probability of LOS is related to the position of the AUs such as their horizontal distances, altitudes, and nearby environments $[18,19]$. Although AUs experience an antenna gain reduction caused by the sidelobe of their associated BSs, Ref. [20] showed that the free-space propagation gain from LOS links can compensate the loss.

Nervertheless, strong LOS links in G2A channel often backfire in the multi-cell networks. This is because, as analyzed in [21,22], AUs also establish LOS links with other unintended terrestrial BSs. The measurements in $[23,24]$ showed that AUs could detect up to 25 or more terrestrial BSs. In downlink, this causes severe ICI to the AUs, where neighboring BSs transmit to their own users using the same RB. The ICI of the AUs is anticipated to be much more severe than that of the TUs located at the cell-edge as AUs' links are LOS and more interfering BSs are detected. Due to high level of ICI, Ref. [25] also showed that mobile AUs are in outage half of the time and velocity plays a role in the connectivity performance. In uplink, the opposite happens as AUs generate ICI to neighboring TUs due to their high altitudes.

Various approaches have been investigated to mitigate the ICI. For instance, Refs. [24,26] have examined the application of the conventional interference mitigation techniques, such as directional antenna beam selection, power control, ICI coordination, and coordinated multi-point (CoMP) transmission. In [27], an optimal time, frequency, and power allocation is proposed to mitigate the ICI. Besides, Refs. [28, 29] exploited the existing coverage extension feature, where this feature relies on repetition coding to achieve a higher maximum coupling loss, higher signal energy, and ultimately mitigate the interference. Consequently, this feature provides more reliability to the AUs' cell-acquisition and supports better mobility.

Furthermore, Ref. [30] showed that power based user association can improve the signal-to-interferenceplus-noise ratio (SINR) and downtilting reduces the ICI, and Ref. [31] showed that using directional antenna with fixed beamwidth at the AUs leads to a tradeoff between the coverage and the ICI level. Moreover, the use of a tilted directional antenna with a fixed beamwidth has also been explored in [32], and a new cooperative beamforming and interference cancellation (CB-ITC) technique has also been proposed in [33]. Nevertheless, the use of a tilted antenna with fixed beamwidth is only beneficial for a low or intermediate network density [32], and CB-ITC can only be employed for low user density [33].

In addition, Refs. [34-36] have explored the use of massive multiple-input-multiple-output (MIMO) for AUs. Specifically, Ref. [34] showed that massive MIMO is a promising strategy as it provides coverage enhancement, spatial multiplexing, and supports high mobility. In [35], the performance of massive MIMO with multiple-users mode and conventional cellular networks with single-user mode are compared. It is further shown in [35] that the earlier outperforms the latter in terms of cumulative distribution function of meeting the AUs' C\&C rate requirement. Relatively, Ref. [36] showed that the use of beamforming improves the performance of the AUs.

It is also worth pointing out that some studies have investigated the AUs' trajectories to mitigate several issues. For instance, Ref. [37] employed reinforcement learning to minimize the trajectory distance, transmission delay, and ICI. Refs. [38,39] optimized the AU's trajectory to reduce outage duration. Besides, Ref. [40] optimized the trajectory to minimize the energy consumption, and Ref. [41] optimized the trajectory to improve the AU computation performance by computational offloading. Other issues such as latency [42] and security risk [43] have also been considered.

Although some research efforts have been given to cellular-connected UAVs, most of the studies (e.g., [20-43]) rely on OMA to support both AUs and TUs. Nevertheless, recent studies have shown that NOMA provides a higher performance gain as compared to conventional OMA. Thanks to the advancement in processor capabilities, the implementation of NOMA is now more practical as well [44].

The use of NOMA for cellular-connected UAVs has received very few attentions as compared to OMA. For example, Ref. [12] proposed a cooperative NOMA scheme that exploits the GS backhaul links for SIC operations, Ref. [45] proposed a transmission technique that incorporates NOMA and zero-forcing beamforming to mitigate the interference, and Ref. [46] proposed an uplink NOMA to serve GU and AU, where AU trajectory and its cell-association order are optimized. Besides, Ref. [47] studied the use of uplink NOMA for AUs and TUs via stochastic geometry, where the mobility of the AUs is taken into account, and Ref. [13] studied the optimal precoding that maximizes the uplink sum-rate of an AU and 
Table 1 Summary of related work

\begin{tabular}{|c|c|c|c|}
\hline Reference & System & Work type & Key findings \\
\hline$[14-17,20-25]$ & OMA & $\begin{array}{l}\text { G2A channel: analysis, measurement, } \\
\text { simulation, modeling }\end{array}$ & $\begin{array}{l}\text { Effects of LOS/NLOS, sidelobe, coverage holes, } \\
\text { shadowing, small-scale fading, interference, mobility }\end{array}$ \\
\hline$[18,19]$ & OMA & $\begin{array}{l}\text { Air-to-ground (A2G) channel: analysis, } \\
\text { measurement, simulation, modeling }\end{array}$ & Probability of LOS/NLOS \\
\hline$[24,26-33]$ & OMA & ICI: analysis and mitigation methods & $\begin{array}{l}\text { Coding, user association, transmitting, } \\
\text { and receiving strategies could efficiently mitigate ICI }\end{array}$ \\
\hline$[34-36]$ & OMA & Massive MIMO & Array gain and spatial multiplexing can be obtained \\
\hline$[37-43]$ & OMA & AUs' trajectories & $\begin{array}{l}\text { AUs may plan their optimal trajectories } \\
\text { based on existing cellular infrastructure }\end{array}$ \\
\hline$[12,13,45-47]$ & NOMA & Uplink & NOMA technique can further be used to \\
\hline$[9,48,49]$ & & Downlink & improve AUs' performance \\
\hline
\end{tabular}

TUs. Nevertheless, Refs. [12,13,45-47] only focus on the uplink communication. In downlink, Ref. [9] proposed a robust NOMA scheme for mobile AU and TU. In [48], NOMA outage for the co-existence of AU and TUs in a single-cell is derived, and Ref. [49] proposed a downlink cooperative NOMA scheme to cancel the ICI via SIC in ideal two-cell networks. As observed, research efforts in NOMA for cellular-connected UAVs are lacking. Hence, we discuss the opportunities and challenges of NOMA for cellular-connected UAVs. The above studies are summarized in Table 1.

\section{Opportunities and challenges for cellular-connected UAVs}

According to [50], NOMA is an optimal scheme in broadcast and medium access channel. In MIMO broadcast and interfering channel, Refs. [51-54] have also shown that NOMA outperforms OMA. Thus, it is promising to employ NOMA for cellular-connected UAVs as to push forward the communication advancement. Furthermore, NOMA provides many other benefits as compared to OMA.

\subsection{Opportunities}

In uplink, AUs and TUs tend to have massive data demands, where these demands may be latencysensitive. To obtain the maximum throughput, OMA strategy is the best. That is, in NOMA, all the power allocation is given to the strong user. Nevertheless, in cases where the RBs are insufficient to serve all users orthogonally, NOMA becomes an outstanding solution. Specifically, NOMA can opportunistically balance the tradeoff between system throughput, latency, and user fairness. As such, latency requirements can be met reliably by many users, while high spectral efficiency can be ensured. Furthermore, AUs tend to have a larger macro-diversity. The large macro diversity can be potentially exploited to achieve a higher performance.

In downlink, AUs and TUs experience asymmetric channel and rate demands. In particular, AUs might experience a stronger channel than that of the TUs. Meanwhile, AUs typically require a lower data rate, whereas TUs require a higher data rate. This asymmetricity is suitable in NOMA because a slight increase of power allocation for the strong user can improve its rate significantly, whereas the rate of the weak user only suffers marginally. This effect can be seen in Figure 1(a), where the power coefficient varies from 0 to 1 , and the linearly-spaced power coefficients at the boundary are drawn. Figure 1(b) further shows NOMA gain over conventional FDMA with opportunistic power allocation, where $\xi$ represents the channel gain difference between AU and TU. Here, $-3 \mathrm{~dB}$ implies that AU's channel gain is twice higher than TU's channel gain, whereas $3 \mathrm{~dB}$ implies the opposite order. Such an observation alludes that NOMA could serve the TUs' links without significant degradation and provides reliable communication to the AUs.

\subsection{Challenges}

In the following, we describe some of the new research challenges in NOMA for cellular-connected UAVs. 


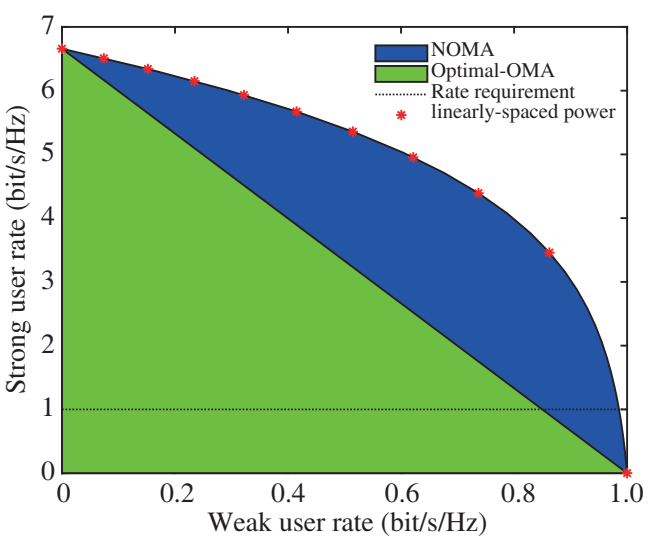

(a)

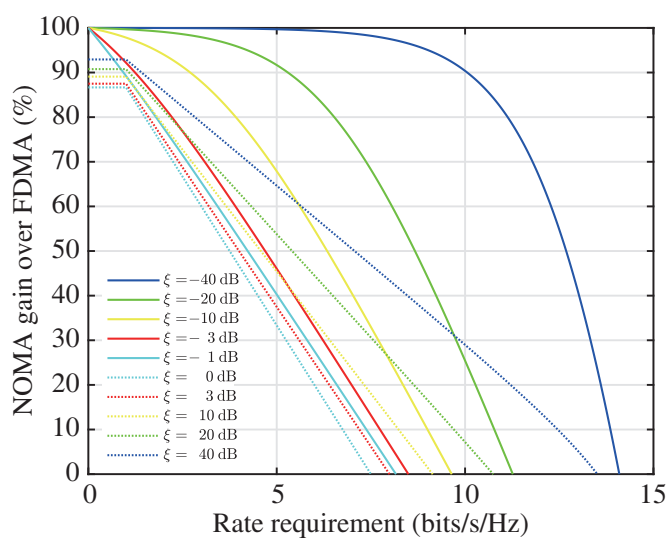

(b)

Figure 1 (Color online) Superiority of NOMA over OMA. (a) Rate region; (b) gain over FDMA.

\subsubsection{SIC order and imperfect SIC for cellular-connected UAVs}

In recent years, several SIC orders have been proposed for terrestrial networks. For instance, there are channel state information (CSI)-based SIC order, QoS-based SIC order [55], and recently [56] proposes a hybrid-SIC order. These SIC orders can be applied to different use-cases. Nevertheless, it remains unclear which SIC orders are beneficial to the applications of NOMA for cellular-connected UAVs. Depending on the use-cases, different SIC orders might have different advantages and disadvantages. Nonetheless, as compared to the terrestrial networks, the impact of SIC for cellular-connected UAVs does not only remain in its own cell or the networks only. For example, in uplink, improper SIC order may engender a stronger ICI level to TUs in neighboring cells. In downlink, imperfect SIC at the AUs may lead to catastrophic events, such as infrastructure damages or loss of lives.

Furthermore, when perfect CSI is unavailable at the BSs, robust schemes that are designed for terrestrial networks may not work well for cellular-connected UAVs. For instance, distance-based ranking [57], which is typically used for terrestrial network to distinguish the strong users and weak users, is no longer accurate $[9,47]$. This is because, given the same horizontal distance, AUs that are hovering at an optimal altitude has the strongest channel gain when compared to the AUs hovering at a lower (i.e., shorter distance) or higher altitude (i.e., longer distance).

Moreover, for cellular-connected UAVs, classifying based on the types of the links as done in terrestrial vehicular networks (e.g., [58]) might not be applicable. This is because the AUs' altitudes and horizontal distances affect their probabilities of LOS. For the same AUs' and TUs' locations, the BS-to-AU links might be stronger or weaker than that of the BS-to-TU links depending on the AUs' link type (e.g., LOS/NLOS). Based on the degree of LOS, the characteristics of large-scale (e.g., path loss exponent) and small-scale (e.g., mean) fading may change significantly as well [9,59].

In addition, SIC may not be ideal in practice. In downlink, the decoded signal of the strong user may be a distorted version of the weak user signal [60-62]. In uplink, the decoded signal of the weak user may be a distorted version of the strong user. Such an imperfect SIC will then lead to error propagation. The rate and outage may thus be compromised. Hence, the impact of SIC orders and imperfect SIC for cellular-connected UAVs remains unclear.

\subsubsection{Power allocation for cellular-connected UAVs}

Due to the aerial propagation, power allocation plays an important role in cellular-connected UAVs. For example, in uplink, the AUs' trajectories and their transmit power would severely affect the ICI level of the TUs in the neighboring cells. Meanwhile, in downlink, the increase of transmit power significantly affect multiple AUs in the sky. Nevertheless, due to the terrestrial propagation (e.g., higher path loss exponent/multipath fading), the increase of transmit power will have a lower impact on the TUs. As a result, careful design on the power allocation is essential for cellular-connected UAVs.

Optimal power allocation has been extensively studied for terrestrial networks. For instance, Refs. [63-65] have presented the optimal power allocation for a large class of objective functions. Given the availability of perfect CSI, the solutions can be easily computed or even expressed in closed-form. Furthermore, the optimal power allocation schemes based on partial CSI have also been investigated 
in $[58,66,67]$.

At the first thought, existing solutions can be applied directly to cellular-connected UAVs. Indeed, if perfect CSI is available, the applications are rather straightforward. Nonetheless, deriving the optimal power allocation based on partial CSI might not be as straightforward as in the cases for terrestrial networks. This is because, as the signals of AUs and TUs are superimposed over a RB, one might need to independently consider the aerial and terrestrial channel statistics to derive the optimal power allocation. Specifically, AUs operating in the sky experience many different effects such as non-stationary channel, strong ICI, mainlobe/sidelobe gains, LOS/NLOS links, and holes in the sky effect [14,20]. These dynamic effects make the channel and interference statistics much more challenging than that of the terrestrial cases.

\subsubsection{User pairing for cellular-connected UAVs}

According to [55], it is not realistic for all users to perform NOMA. Instead, a promising alternative is to employ a multi-carrier NOMA (MC-NOMA) system, where the users are divided into multiple groups. Each group is then being allocated orthogonal RBs and users in each group performs NOMA. The performance of MC-NOMA system depends on how users are grouped together. Hence, many studies (e.g., [68-74]) have investigated the user pairing problem for terrestrial networks, and pointed out the computational complexity as a major bottleneck.

Nonetheless, cellular-connected UAVs introduce more challenges to user pairing problem because, unlike TUs, AUs are more likely to experience frequent handover, severe ICI, temporary non-LOS, and holes in the sky effects. To ensure optimal performance, the pairing decision has to be quick and robust. Besides, AUs and TUs experience different propagation, and have different characteristics and demands. The pairing designs and concerns may thus be very different from the conventional case.

For example, in downlink, one might want to pair AU and TU together as this pairing could serve TU link as usual and provide an additional connectivity to AU. On the contrary, in uplink, one might want to pair AUs together so that multiple BSs could perform cooperative SIC to enhance their performance. Due to its combinatorial nature, pairing of multiple AUs and TUs in a group remains to be a challenging yet interesting issue to be explored.

\subsubsection{Intercell interference mitigation for cellular-connected UAVs}

Using NOMA in terrestrial networks is also challenged by the ICI, but mainly for the cell-edge users. As discussed in [75], several CoMP techniques have been proposed to enhance NOMA performance for celledge users. For instance, a coordinated scheduling (CS)-NOMA scheme based on fractional power ratio has been proposed in [76], Alamouti code in NOMA is also proposed in [77], coordinated beamforming (CB)-NOMA has also been adopted in [78], and joint transmission (JT)-NOMA technique, also known as the network NOMA has also been investigated in [79]. These schemes can be adopted for cellularconnected UAVs.

Nevertheless, in downlink, the ICI level of the AUs is anticipated to be much higher than that of the cell-edge TUs due to their high altitudes and LOS links. Therefore, conventional coordination among adjacent BSs is insufficient to mitigate the ICI at the AUs. To completely cancel the ICI, coordination and information exchanged among larger number of BSs are required. Such solutions render high complexity and backhaul congestion as AUs could detect up to tens of BSs. Clearly, coordination among limited number of BSs to efficiently mitigate the ICI of the AUs is desired and such issue remains to be addressed.

In uplink, AUs generate strong ICI to the TUs. Although cooperative SIC can be performed by the BSs to reduce the ICI, this solution may also render backhaul congestion. In particular, AUs might transmit tens of gigabit per second (Gbps) of data in the uplink. To successfully perform SIC, a large fraction of this data has to be delivered to other BSs. The backhaul links may thus be overwhelmed with AUs' uplink data. To overcome this limitation, precoding can be employed. However, this solution requires multiple antennas at the AUs.

\subsubsection{MIMO-NOMA for cellular-connected UAVs}

MIMO technology is a promising solution as it provides high spectral efficiency and reliability through spatial multiplexing and diversity gains. Therefore, it is appealing to employ MIMO for cellular-connected UAVs. To obtain high spatial multiplexing and diversity gains for aerial channel, both antenna separation 


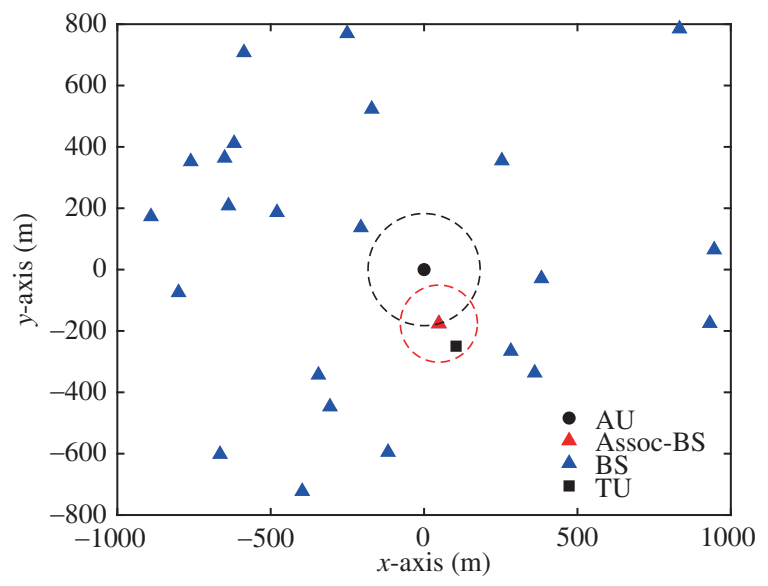

Figure 2 (Color online) Downlink multi-cell networks with co-existence of AU and TU.

and orientation have to be carefully chosen. Unlike TUs, certain selections are not practical on AUs due to their shapes, sizes and weight constraints. Besides, MIMO incurs higher system complexity and power consumption. Therefore, the number of antennas, radio-frequency chains, and type of architecture have to be carefully designed.

When multiple antennas are well-implemented at the AUs, MIMO-NOMA can be adopted. Several MIMO-NOMA schemes have been proposed for terrestrial networks. For instance, Refs. [80] proposed a detection vector to avoid inter-cluster interference. This work is further extended in [81] by exploiting the concept of signal-alignment. Furthermore, NOMA can also be implemented in massive-MIMO system $[82,83]$. These schemes can be similarly adopted for cellular-connected UAVs. Nevertheless, due to $3 \mathrm{D}$ mobility, which results in rapid varying aerial channel, obtaining accurate CSI becomes a key challenge (see Subsection 3.2.2). Refs. [84] and [85] further show that partial CSI leads to limited spatial multiplexing and diversity gains, respectively. In NOMA, inaccurate CSI also affects the rate and reliability of the paired users.

Besides, in some cases, the feasibility of MIMO-NOMA for cellular-connected UAVs remains unclear. For instance, AUs are scattered around the sky and TUs are scattered around the ground. Although their channel properties are very different, they might have a degree of correlation. Thus, it is an interesting issue whether MIMO-NOMA, in practice, can serve these receivers in a group and if MIMO-NOMA continues to outperform MIMO-OMA in these cases.

\section{Simulation details}

In this section, we provide the details of our Monte-Carlo simulation. The aim of this simulation is to conceptually analyze the AU's signal characteristics and evaluate the performance of NOMA for the co-existence of AU and TU.

\subsection{Simulation framework}

As depicted in Figure 2, we consider a downlink wireless communication system with multiple terrestrial $\mathrm{BSs}$, a TU, and an AU. In this simulation, we assume the BSs are distributed according to a homogeneous poisson point process $(\mathrm{HPPP}) \Phi_{\mathcal{B}}$ with a fixed height of $z_{b}$ and intensity $\lambda_{b}$. The AU is assumed at the origin with fixed altitude of $z_{u}$.

We denote $b_{0}$ as the nearest $\mathrm{BS}$ to the $\mathrm{AU}$ and $r_{0}$ as the horizontal distance from the AU to BS $b_{0}$. Furthermore, we denote $r_{1}$ as the nearest distance from BS $b_{0}$ to $\mathrm{BS} b \in \Phi_{\mathcal{B}} /\left\{b_{0}\right\}$. The TU is then distributed uniformly around the $\mathrm{BS} b_{0}$ with a radius $\frac{r_{1}}{2}$, and a fixed height of $z_{t}$, where $z_{u} \gg z_{t}$. We then assume the AU and TU are associated to BS $b_{0}$.

The aerial communication link between the AU and the BS $b$ is modeled using the probability LOS /NLOS model as in [86]. In this model, there are two types of links, $v \in\{\mathrm{L}, \mathrm{N}\}$, where $\mathrm{L}$ and $\mathrm{N}$ represent the LOS and NLOS links, respectively. The probability of LOS between the AU and BS $b$ is

$$
p_{b, u}^{\mathrm{L}}\left(\phi_{b, u}\right)=-\varphi \cdot \exp \left(-\xi \cdot \phi_{b, u}\right)+\zeta
$$


where $\phi_{b, u}$ is the elevation angle between the $\mathrm{BS} b$ and $\mathrm{AU}$, and $\varphi, \xi$, and $\zeta$ are constant coefficients related to the communication environment as given in [86].

Given link type $v$, the channel gain between the $\mathrm{AU}$ and $\mathrm{BS} b$ is

$$
\left|h_{b, u}^{v}\right|^{2}=\Xi_{b, u}^{v}\left|\Omega_{b, u}^{v}\right|^{2}, \quad v \in\{\mathrm{L}, \mathrm{N}\}
$$

where $\Xi_{b, u}^{v}$ and $\left|\Omega_{b, u}^{v}\right|^{2}$ are the large and small-scale fading effects, respectively. In the large-scale fading, $\Xi_{b, u}^{v} \triangleq \frac{E_{u} F G_{u}^{\mathrm{rx}}}{r_{b, u} \alpha_{v}}$, where $E_{u}$ is the sidelobe gain from the BS, $F=\left(\frac{4 \pi f_{c}}{c}\right)^{-2}$ is the attenuation loss at the operating frequency $f_{\mathrm{c}}, G_{u}^{\mathrm{rx}}$ is the receiving antenna gain at the $\mathrm{AU}, \alpha_{v}$ denotes the aerial path loss exponents. In the small scale fading, $\left|\Omega_{b, u}^{v}\right|^{2} \sim \operatorname{Gamma}\left(m_{u}^{v}, \theta_{u}^{v}\right)$.

Meanwhile, the terrestrial communication link between the TU and BS $b$ follows the conventional modeling. Specifically, the channel gain between the TU and BS $b$ is

$$
\left|h_{b, t}\right|^{2}=\Xi_{b, t}\left|\Omega_{b, t}\right|^{2} .
$$

Here, $\Xi_{b, t}$ and $\left|\Omega_{b, t}\right|^{2}$ are similarly the large and small-scale fading effects, respectively. Nevertheless, in (3), the large scale fading is defined as $\Xi_{b, t} \triangleq \frac{E_{t} F G_{t}^{\text {rx }}}{r_{b, t^{\alpha}}}$, where $E_{t}$ accounts for the mainlobe gain from the $\mathrm{BS}, G_{t}^{\mathrm{rx}}$ is the receiving antenna gain at the TU, and $\alpha$ is the terrestrial path loss exponent. In the small scale fading, $\left|\Omega_{b, t}\right|^{2} \sim \operatorname{Gamma}\left(m_{t}, \theta_{t}\right)$.

Furthermore, we assume NOMA is employed to serve the AU and TU concurrently. To ensure high reliability, AU decodes its message directly with the following SINR:

$$
\operatorname{SINR}_{u}^{v}=\frac{\rho_{u} P_{\mathrm{tx}}\left|h_{b_{0}, u}^{v}\right|^{2}}{\rho_{t} P_{\mathrm{tx}}\left|h_{b_{0}, u}^{v}\right|^{2}+\mu \sum_{b \in \Phi_{\mathcal{B}} /\left\{b_{0}\right\}} P_{\mathrm{tx}}\left|h_{b, u}^{v}\right|^{2}+N_{0}},
$$

where $P_{\mathrm{tx}}$ is the transmission power of BS, $N_{0}$ is the noise power level, and $\rho_{u}$ and $\rho_{t}$ are the power coefficient for $\mathrm{AU}$ and $\mathrm{TU}$, respectively. In (4), $\mu \in[0,1]$ is an auxiliary variable, where this variable is only used to analyze the ICI effect. In more realistic cases, $\mu$ depends on the efficiency of the ICI mitigation techniques. Efficient and practical ICI mitigation techniques for cellular-connected UAVs are clearly desirable. The achievable rate of AU is

$$
R_{u}=B_{\mathrm{w}} \log \left(1+\operatorname{SINR}_{u}^{v}\right) .
$$

Following NOMA principle, we assume the TU performs SIC by first removing the message of the AU. Then, the TU decodes its own message with the following SINR:

$$
\operatorname{SINR}_{t}=\frac{\rho_{t} P_{\mathrm{tx}}\left|h_{b_{0}, t}\right|^{2}}{\sum_{b \in \Phi_{\mathcal{B}} /\left\{b_{0}\right\}} P_{\mathrm{tx}}\left|h_{b, t}\right|^{2}+N_{0}} .
$$

The achievable rate of $\mathrm{TU}$ is therefore

$$
R_{t}=B_{\mathrm{w}} \log \left(1+\mathrm{SINR}_{t}\right) .
$$

The simulation parameters used in this article are shown in Table 2.

\subsection{Performance metrics}

We evaluate the user performance using two metrics: coverage probability and average user rate. The coverage probability is defined as

$$
\mathcal{P}_{\text {cov }}=\mathbb{P}(\operatorname{SINR}>T),
$$

where SINR denotes the SINR of the AU/TU, i.e., (4) and (6). The average user rate is

$$
\mathcal{R}=\mathbb{E}\left[B_{\mathrm{w}} \log (1+\mathrm{SINR})\right] .
$$


Table 2 Simulation parameters

\begin{tabular}{cccc}
\hline Parameter & Value & Parameter & Value \\
\hline$(\varphi, \xi, \zeta)$ & $(1,0.151,1)$ & $G_{u}^{\mathrm{rx}} / G_{t}^{\mathrm{rx}}$ & $1 \mathrm{~dB}$ \\
$\left(E_{u}, E_{t}\right)$ & $(-3,10) \mathrm{dB}$ & $\left(m_{u}^{\mathrm{L}}, m_{u}^{\mathrm{N}}, m_{t}\right)$ & $(3,2,1)$ \\
$\lambda_{b}$ & $10 \mathrm{BSs} / \mathrm{km}^{2}$ & $\left(\theta_{u}^{\mathrm{L}}, \theta_{u}^{\mathrm{N}}, \theta_{t}\right)$ & $\left(\frac{1}{3}, \frac{1}{2}, 1\right)$ \\
$B_{\mathrm{w}}$ & $180 \mathrm{KHz} / \mathrm{RB}$ & $f_{\mathrm{c}}$ & $2 \mathrm{GHz}$ \\
\hline
\end{tabular}

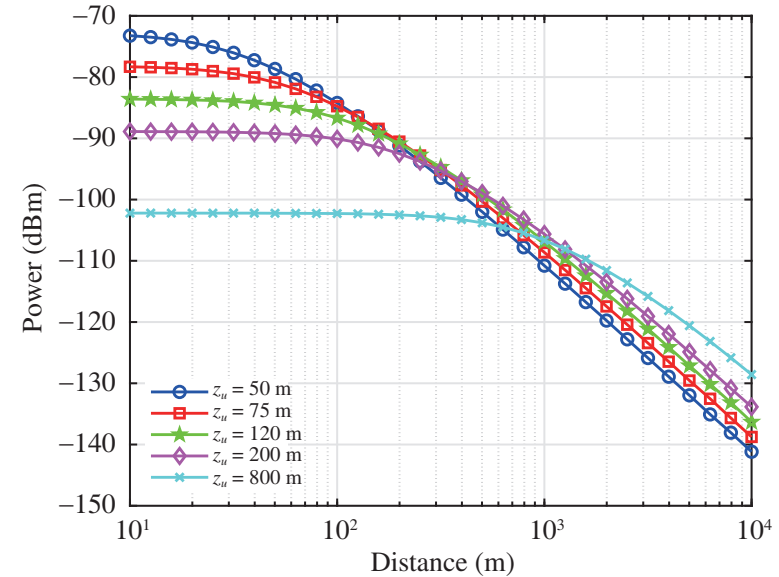

(a)

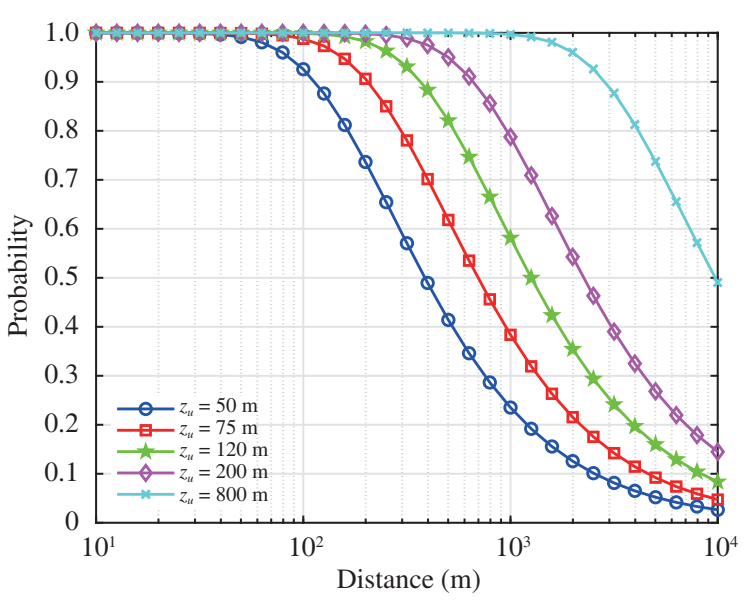

(b)

Figure 3 (Color online) Ground-to-air channel. (a) Channel attenuation; (b) probability of LOS.

\section{Result discussion}

\subsection{Ground-to-air channel characteristics}

Figure 3 shows the G2A channel characteristics w.r.t. AU's horizontal distance. Five altitudes are considered: 50, 75, 120, 200, and $800 \mathrm{~m}$. As seen in Figure 3(a), the channel attenuates with the increase of AU's horizontal distance. When the distance is small, the channel attenuates slowly due to the path loss effect. However, when the distance is large, the channel at a lower altitude attenuates more rapidly as compared to the channel at a higher altitude. This effect is due to the probability of LOS. As shown in Figure 3(b), the probability of LOS decreases more rapidly at a lower altitude as compared to a higher altitude. In practice, different applications may require AUs to operate at different altitudes. This suggests that the channel characteristics can be exploited to design efficient transmitting and receiving strategies.

\subsection{AU's signal characteristics}

Figure 4 shows the AU's signal characteristics, where the power coefficient allocated to the AU is $\rho_{u}=1$. As shown in Figure 4(a), the coverage probability is high at a low SINR regime. Nevertheless, it declines steeply beyond a certain threshold. This declination is related to the desired and ICI signal strengths. The variation in declination for different altitudes is related to the G2A characteristics, as discussed in Subsection 5.1 .

In Figure 4(b), it is observed that higher AU's altitude generally improves the desired signal strength due to a higher probability of LOS. Nevertheless, the desired signal strength drops if the AU's altitude exceeds the height where the LOS gain cannot compensate the path loss attenuation. This observation can be similarly seen in Figure 4(c), where the ICI is more severe at a higher altitude. For a fixed BS density, the ICI level is bounded by a certain threshold (e.g., $-55 \mathrm{dBm}$ ). This is because while the probabilities of LOS to interfering BSs are higher, their path loss are also larger. This suggests that the ICI is not going to increase indefinitely. Hence, there is an upper bound. Such an upper bound can be used as a benchmark to evaluate or compare the performance of different transmit and receive schemes.

Besides, the declination of the coverage probability is related to the ICI. For instance, if the slope of the ICI is steep, the declination of the coverage probability is also steep. Therefore, ICI is a major 


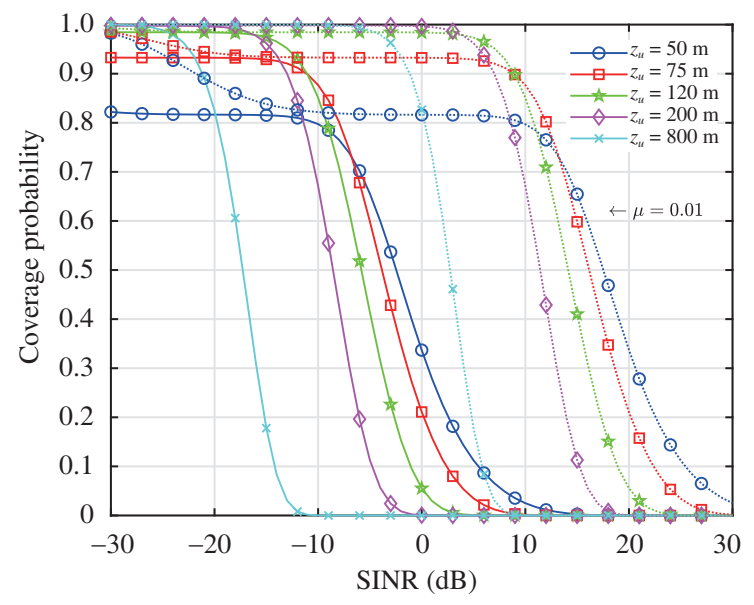

(a)

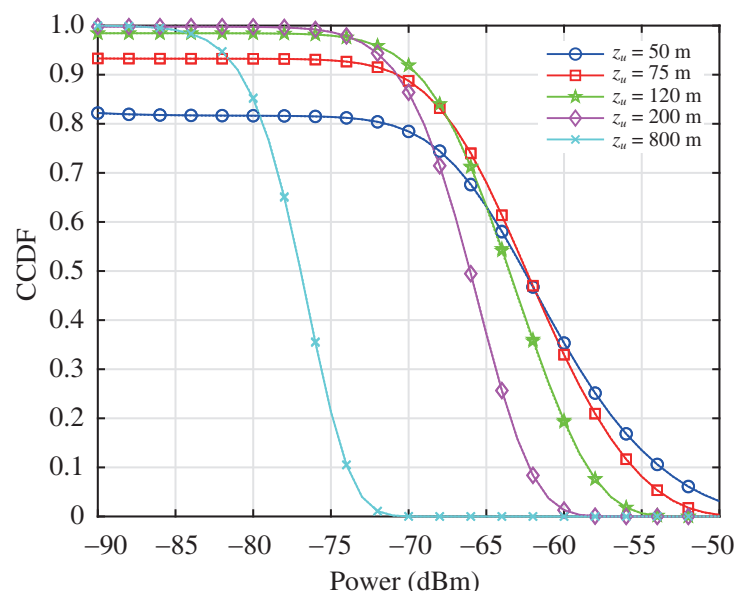

(b)

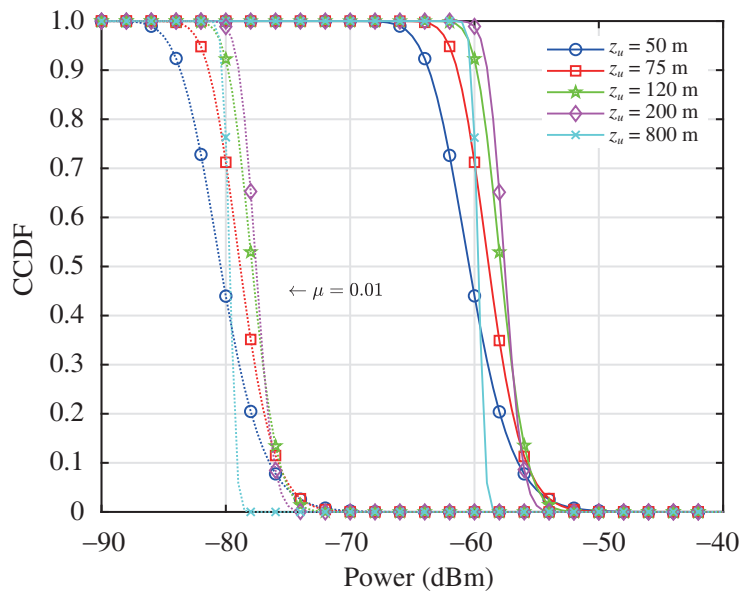

(c)

Figure 4 (Color online) Signal characteristics. (a) Coverage probability; (b) desired signal strength; (c) ICI level.

impediment in providing reliable connectivity to the AU. This motivates us to conceptually investigate how the coverage probability performs when the ICI is mitigated.

According to [21], the rate requirement for AU's C\&C link is $100 \mathrm{Kbps}$, which is equivalent to a threshold of $-3.2 \mathrm{~dB}$ for a $\mathrm{RB}$ of $180 \mathrm{KHz}$ bandwidth. Meanwhile, the reliability requirement is at least 99.9\%. Based on Figure 4, using ICI mitigation techniques are clearly required to reliably serve the AU's $\mathrm{C} \& \mathrm{C}$ link. Here, we make use of the auxiliary variable, $\mu$, to conceptually investigate the opportunity. If $\mu=0.01$, the ICI level is reduced by $20 \mathrm{~dB}$. Since the system is operating in the interference-limited regime, the AU's coverage probability is improved by $20 \mathrm{~dB}$.

As shown in Figure 4(a), if $\mu=0.01$, only the AU's altitudes at 200 and $120 \mathrm{~m}$ achieve the coverage probabilities near to 1 at $-3.2 \mathrm{~dB}$. At $800 \mathrm{~m}$, the AU's coverage probability is slightly lower. In addition, the AU's coverage probabilities at 75 and $50 \mathrm{~m}$ are significantly lower because, as shown in Figure 4(b), the desired signal strength remains low. In contrast, at a high SINR regime, the AU's coverage probabilities at 75 and $50 \mathrm{~m}$ are higher as compared to 800, 200, and $120 \mathrm{~m}$. Hence, our result shows that decreasing the AU's altitude is not always a promising strategy. Instead, to improve the coverage probability at a low SINR regime (e.g., low data rate applications), one may want to keep AU's altitude high, but not exceeding the point where the path loss becomes dominant and larger than LOS gain. On the contrary, one may want to keep AU's altitude low to improve the coverage probability at a high SINR regime (e.g., high data rate applications).

Based on these results, it is counter intuitive to employ NOMA because it introduces interference. Nevertheless, our investigation shows an interesting result. Figure 5 shows the AU's signal characteristics with the presence of NOMA co-channel interference. The power coefficients assigned to the AU and TU are $\rho_{u}=0.4$ and $\rho_{t}=0.6$, respectively, and $\mu=0.01$. Indeed, at a high SINR regime, the coverage probability 


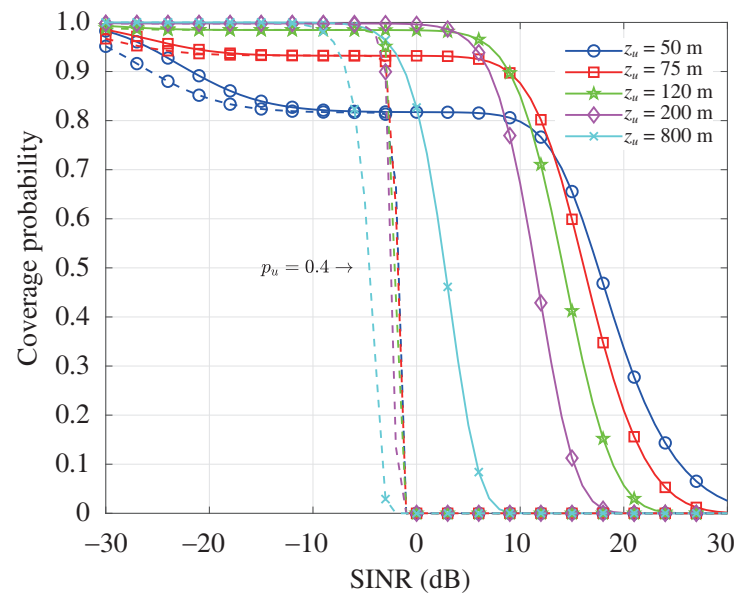

(a)

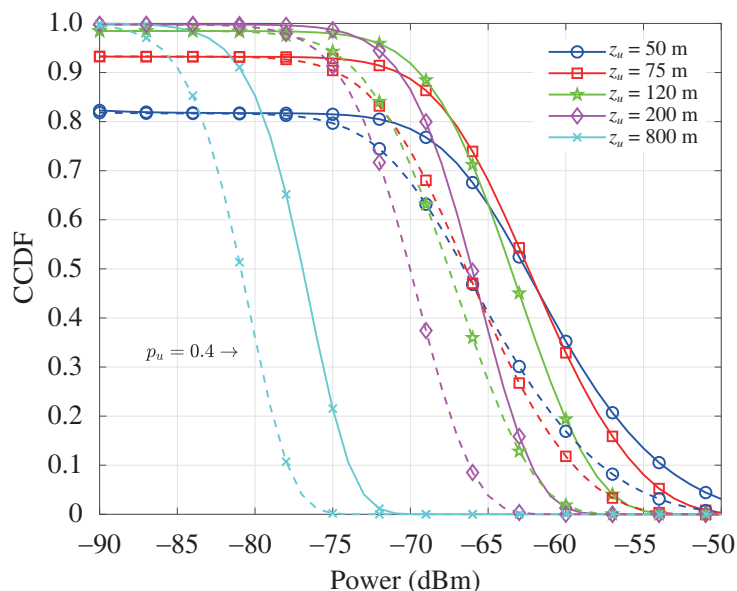

(b)

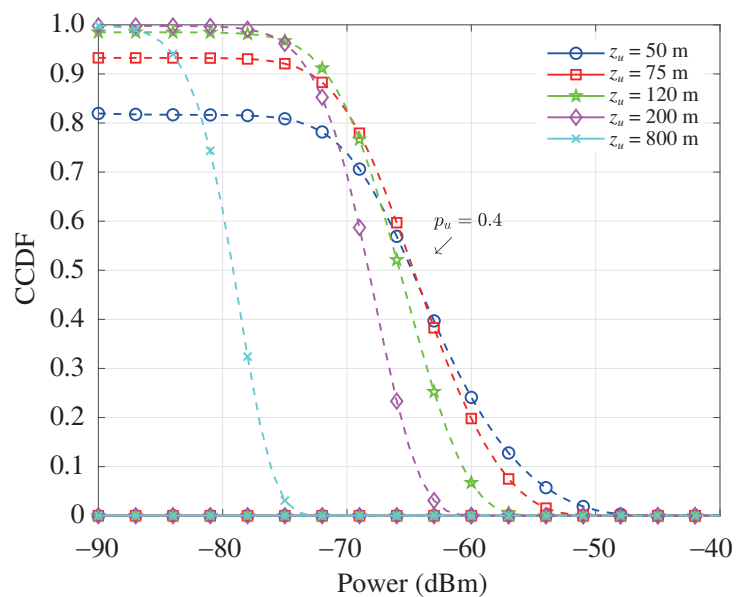

(c)

Figure 5 (Color online) Signal behaviors. (a) Coverage probability; (b) desired signal; (c) NOMA co-channel interference.

is degraded. Nevertheless, at a low SINR regime, the coverage probability is not much affected. As shown in (4), this occurs because the SINR is determined by the power coefficients, and the channel gain between the AU and its associated BS. For instance, as seen in Figure 5(b), the desired signal is reduced if $\rho_{u}=0.4$. Meanwhile, Figure 5(c) shows the strength of NOMA co-channel interference increases if $\rho_{t}=0.6$. Therefore, the coverage probability at a low SINR regime is not significantly affected if the power coefficients are chosen appropriately. In fact, NOMA can have a comparable coverage probability to the settings of $\rho_{u}=1$ and $\mu=0.01$ at a low SINR regime. It is worth mentioning that the system does not need to operate in noise-limited regime to achieve this performance. Instead, this performance can be achieved when the desired signal strength is stronger than the ICI level. Otherwise, the ICI of the AU remains to be the limiting factor.

\subsection{NOMA versus OMA}

Figure 6 shows the average user rate. Here, we compare NOMA and OMA schemes. For the latter, we consider FDMA with equal bandwidth allocation. In OMA, AU is allocated with an orthogonal RB. Thus, unlike NOMA, AU does not suffer any co-channel interference from the TU's signal. Nevertheless, AU still suffers ICI from other BSs. This is the same in the case of NOMA. In NOMA, we consider two sets of power allocations with: $\rho_{u}=0.9$ and $\rho_{u}=0.6$, where $\rho_{t}=1-\rho_{u}$. In both schemes, we also consider the cases where $\mu=1$ and $\mu=0.01$.

As shown in Figure 6(a), NOMA generally outperforms OMA in terms of the TU rate. Due to the fixed power allocation, the average TU rate is independent from the AU's altitude and $\mu$. Nevertheless, in NOMA, we can see that the average TU rate is significantly affected by $\rho_{u}$. Thus, if the AU only requires a low data rate, one may allocate a larger $\rho_{t}$ to improve the TU rate. 


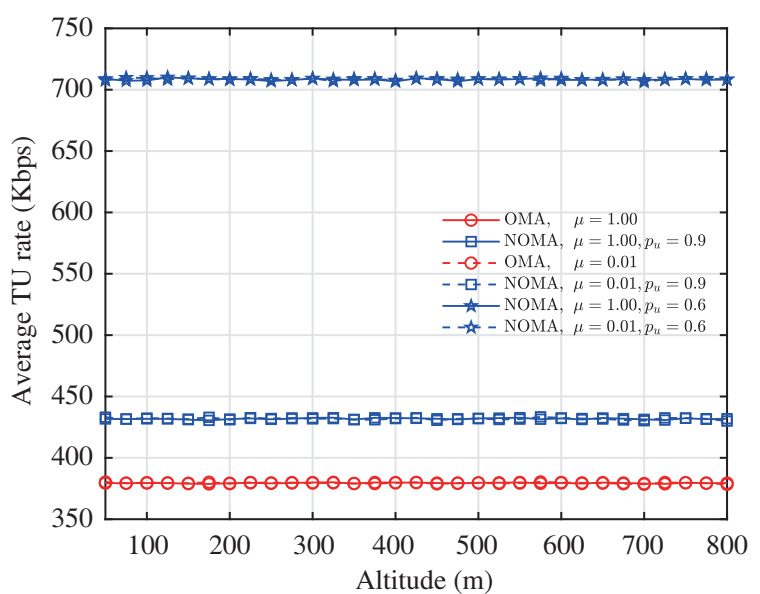

(a)

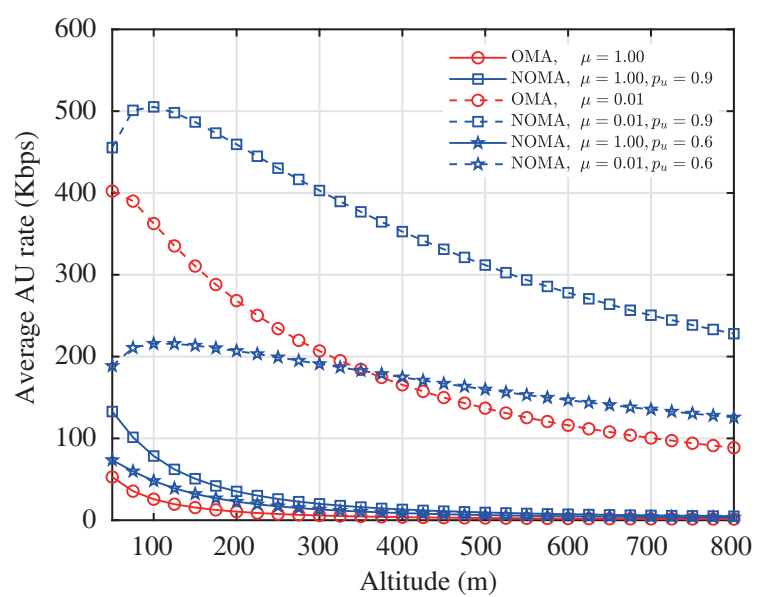

(b)

Figure 6 (Color online) NOMA vs. OMA. (a) Average TU rate; (b) average AU rate.

Similarly, in Figure 6(b), NOMA generally outperforms OMA in terms of the AU rate for the same $\mu$. Only for the case where $\mu=0.01$ and $\rho_{u}=0.6$, we can see that OMA outperforms NOMA if the AU's altitude is below $350 \mathrm{~m}$. This is because, in our simulation, we adopt a fixed SIC order, where AU always decodes the signal directly and TU always performs SIC. Therefore, if AU has a stronger channel than that of TU, NOMA suffers a significant performance degradation [9].

To address this limitation, there are at least two methods. Firstly, one may adopt an adaptive SIC order to guarantee the superiority of NOMA over OMA. Secondly, one may choose an appropriate power allocation to outperform OMA. The latter can be observed in the case where $\mu=0.01$ and $\rho_{u}=0.9$.

\section{Conclusion}

In this paper, we have discussed the applications of NOMA for cellular-connected UAVs. To this end, we have reviewed important findings of the related works. New research opportunities and challenges in NOMA for cellular-connected UAVs are also outlined. Furthermore, we have conceptually studied downlink NOMA for cellular-connected UAVs, where both AU and TU are present. In particular, MonteCarlo simulations are performed to analyze the new AUs' signal characteristics and to evaluate the performance of downlink NOMA for co-existence of AU and TU. The simulation results highlight a set of opportunities and challenges. Based on the results, we conclude that NOMA is an appealing solution for cellular-connected UAVs as it supports higher connectivity and may further improve the average user rate performance as compared to the OMA scheme. Acknowledgements The work of Z G Ding was supported by the UK EPSRC (Grant No. EP/P009719/2). This work was also
supported in part by H2020-MSCA-RISE-2015 (Grant No. 690750) and Ministry of Education Malaysia and Universiti Teknologi
Malaysia (Grant Nos. 4J416, 08G83, 19H58, 04G37).

Open access This article is licensed under a Creative Commons Attribution 4.0 International License, which permits use, sharing, adaptation, distribution and reproduction in any medium or format, as long as you give appropriate credit to the original author(s) and the source, provide a link to the Creative Commons licence, and indicate if changes were made. The images or other third party material in this article are included in the article's Creative Commons licence, unless indicated otherwise in a credit line to the material. If material is not included in the article's Creative Commons licence and your intended use is not permitted by statutory regulation or exceeds the permitted use, you will need to obtain permission directly from the copyright holder. To view a copy of this licence, visit http://creativecommons.org/licenses/by/4.0/.

\section{References}

1 Hayat S, Yanmaz E, Muzaffar R. Survey on unmanned aerial vehicle networks for civil applications: a communications viewpoint. IEEE Commun Surv Tut, 2016, 18: 2624-2661

2 Zeng Y, Lyu J B, Zhang R. Cellular-connected UAV: potential, challenges, and promising technologies. IEEE Wirel Commun, 2019, 26: 120-127

3 Zeng Y, Wu Q Q, Zhang R. Accessing from the sky: a tutorial on UAV communications for 5G and beyond. Proc IEEE, 2019, 107: $2327-2375$

4 Afonso L, Souto N, Sebastiao P, et al. Cellular for the skies: exploiting mobile network infrastructure for low altitude air-to-ground communications. IEEE Aerosp Electron Syst Mag, 2016, 31: 4-11

5 Goddemeier N, Daniel K, Wietfeld C. Coverage evaluation of wireless networks for unmanned aerial systems. In: Proceedings of IEEE Globecom Workshops, Miami, 2010. 1760-1765 
6 Dai L L, Wang B C, Ding Z G, et al. A survey of non-orthogonal multiple access for 5G. IEEE Commun Surv Tut, 2018, 20: $2294-2323$

7 Ding Z G, Lei X F, Karagiannidis G K, et al. A survey on non-orthogonal multiple access for 5 G networks: research challenges and future trends. IEEE J Sel Areas Commun, 2017, 35: 2181-2195

8 Tse D, Viswanath P. Fundamentals of Wireless Communication. Cambridge: Cambridge University Press, 2005

9 New W K, Leow C Y, Navaie K, et al. Robust non-orthogonal multiple access for aerial and ground users. IEEE Trans Wirel Commun, 2020, 19: 4793-4805

10 Agiwal M, Roy A, Saxena N. Next generation 5G wireless networks: a comprehensive survey. IEEE Commun Surv Tut, 2016, 18: $1617-1655$

11 Generation Partnership Project. Unmanned aerial system (UAS) support in 3GPP — stage 1. TS 22.125. 2019

12 Mei W D, Zhang R. Uplink cooperative NOMA for cellular-connected UAV. IEEE J Sel Top Signal Process, 2019, 13: 644-656

13 Pang X W, Gui G, Zhao N, et al. Uplink precoding optimization for NOMA cellular-connected UAV networks. IEEE Trans Commun, 2020, 68: 1271-1283

14 Teng E, Falcao J, Iannucci B. Holes-in-the-sky: a field study on cellular-connected UAS. In: Proceedings of International Conference on Unmanned Aircraft Systems (ICUAS), Miami, 2017. 1165-1174

15 Khawaja W, Guvenc I, Matolak D W, et al. A survey of air-to-ground propagation channel modeling for unmanned aerial vehicles. IEEE Commun Surv Tut, 2019, 21: 2361-2391

16 Khuwaja A A, Chen Y F, Zhao N, et al. A survey of channel modeling for UAV communications. IEEE Commun Surv Tut, 2018, 20: 2804-2821

17 Amorim R, Nguyen H, Mogensen P, et al. Radio channel modeling for UAV communication over cellular networks. IEEE Wirel Commun Lett, 2017, 6: 514-517

18 Al-Hourani A, Kandeepan S, Jamalipour A. Modeling air-to-ground path loss for low altitude platforms in urban environments. In: Proceedings of IEEE Global Communications Conference, Austin, 2014. 2898-2904

19 Al-Hourani A, Gomez K. Modeling cellular-to-UAV path-loss for suburban environments. IEEE Wirel Commun Lett, 2018, 7: $82-85$

20 Lin X Q, Yajnanarayana V, Muruganathan S D, et al. The sky is not the limit: LTE for unmanned aerial vehicles. IEEE Commun Mag, 2018, 56: 204-210

21 Generation Partnership Project. Study on Enhanced LTE Support for Aerial Vehicles. Technical Report 36.777, 2017

22 Qualcomm Technologies, Inc. LTE Unmanned Aircraft Systems: Trial Report v1.0.1. Technical Report, 2017

23 Bergh B V D, Chiumento A, Pollin S. LTE in the sky: trading off propagation benefits with interference costs for aerial nodes. IEEE Commun Mag, 2016, 54: 44-50

24 Kovacs I, Amorim R, Nguyen H C, et al. Interference analysis for UAV connectivity over LTE using aerial radio measurements. In: Proceedings of the 86th Vehicular Technology Conference (VTC-Fall), Toronto, 2017

25 Stanczak J, Kovacs I, Koziol D, et al. Mobility challenges for unmanned aerial vehicles connected to cellular LTE networks. In: Proceedings of the 87th Vehicular Technology Conference (VTC Spring), Porto, 2018

26 Nguyen H C, Amorim R, Wigard J, et al. How to ensure reliable connectivity for aerial vehicles over cellular networks. IEEE Access, 2018, 6: 12304-12317

27 Xue Z, Wang J L, Shi Q J, et al. Time-frequency scheduling and power optimization for reliable multiple UAV communications. IEEE Access, 2018, 6: 3992-4005

28 Yajnanarayana V, Wang Y P E, Gao S W, et al. Interference mitigation methods for unmanned aerial vehicles served by cellular networks. In: Proceedings of IEEE 5G World Forum (5GWF), Silicon Valley, 2018. 118-122

29 Euler S, Maattanen H, Lin X, et al. Mobility support for cellular connected unmanned aerial vehicles: performance and analysis. In: Proceedings of IEEE Wireless Communications and Networking Conference (WCNC), Marrakesh, 2019

$30 \mathrm{Xu}$ X L, Zeng Y. Cellular-connected UAV: performance analysis with 3D antenna modelling. In: Proceedings of IEEE International Conference on Communications Workshops (ICC Workshops), Shanghai, 2019

31 Azari M M, Rosas F, Pollin S. Reshaping cellular networks for the sky: major factors and feasibility. In: Proceedings IEEE International Conference on Communications (ICC), Kansas, 2018

32 Azari M M, Rosas F, Pollin S. Cellular connectivity for UAVs: network modeling, performance analysis, and design guidelines. IEEE Trans Wirel Commun, 2019, 18: 3366-3381

33 Mei W D, Zhang R. Cooperative downlink interference transmission and cancellation for cellular-connected UAV: a divideand-conquer approach. IEEE Trans Commun, 2020, 68: 1297-1311

34 Chandhar P, Larsson E G. Massive MIMO for connectivity with drones: case studies and future directions. IEEE Access, 2019, 7: 94676-94691

35 Geraci G, Garcia-Rodriguez A, Giordano L G, et al. Understanding UAV cellular communications: from existing networks to massive MIMO. IEEE Access, 2018, 6: 67853-67865

36 Amer R, Saad W, Marchetti N. Toward a connected sky: performance of beamforming with down-tilted antennas for ground and UAV user co-existence. IEEE Commun Lett, 2019, 23: 1840-1844

37 Challita U, Saad W, Bettstetter C. Interference management for cellular-connected UAVs: a deep reinforcement learning approach. IEEE Trans Wirel Commun, 2019, 18: 2125-2140

38 Zhang S W, Zhang R. Trajectory design for cellular-connected UAV under outage duration constraint. In: Proceedings of IEEE International Conference on Communications (ICC), Shanghai, 2019

39 Bulut E, Guevenc I. Trajectory optimization for cellular-connected UAVs with disconnectivity constraint. In: Proceedings of IEEE International Conference on Communications Workshops (ICC Workshops), Kansas, 2019

40 Khamidehi B, Sousa E S. Power efficient trajectory optimization for the cellular-connected aerial vehicles. In: Proceedings of the 30th Annual International Symposium on Personal, Indoor and Mobile Radio Communications (PIMRC), Istanbul, 2019

41 Cao X W, Xu J, Zhang R. Mobile edge computing for cellular-connected UAV: computation offloading and trajectory optimization. In: Proceedings of the 19th International Workshop on Signal Processing Advances in Wireless Communications (SPAWC), Kalamata, 2018

42 Long Y, Yang T, Feng H, et al. Latency-aware base station selection scheme for cellular-connected UAVs. In: Proceedings of the 88th Vehicular Technology Conference (VTCFall), Chicago, 2018

43 Challita U, Ferdowsi A, Chen M Z, et al. Machine learning for wireless connectivity and security of cellular-connected UAVs. IEEE Wirel Commun, 2019, 26: 28-35

44 Vaezi M, Ding Z G, Poor H V. Multiple Access Technique for 5G Wireless Networks and Beyond. Berlin: Springer, 2019

45 Liu L, Zhang S W, Zhang R. Exploiting NOMA for multi-beam UAV communication in cellular uplink. In: Proceedings of IEEE International Conference on Communications (ICC), Shanghai, 2019

$46 \mathrm{Mu}$ X D, Liu Y W, Guo L, et al. Non-orthogonal multiple access for air-to-ground communication. IEEE Trans Commun, 2020. doi: 10.1109/TCOMM.2020.2973264 
47 Senadhira N, Durrani S, Zhou X Y, et al. Uplink NOMA for cellular-connected UAV: impact of UAV trajectories and altitude. IEEE Trans Commun, 2020, 68: 5242-5258

48 Zaidi S K, Hasan S F, Gui X. Outage analysis of ground-aerial NOMA with distinct instantaneous channel gain ranking. IEEE Trans Veh Technol, 2019, 68: 10775-10790

49 Mei W D, Zhang R. Cooperative NOMA for downlink asymmetric interference cancellation. IEEE Wirel Commun Lett, 2020, 9: $884-888$

50 El-Gamal A, Kim Y. Networks Information Theory. Cambridge: Cambridge University Press, 2011

51 Zeng M, Yadav A, Dobre O A, et al. Capacity comparison between MIMO-NOMA and MIMO-OMA with multiple users in a cluster. IEEE J Sel Areas Commun, 2017, 35: 2413-2424

52 Liu Y, Pan G F, Zhang H T, et al. On the capacity comparison between MIMO-NOMA and MIMO-OMA. IEEE Access, 2016, 4: $2123-2129$

53 Ali M S, Hossain E, Kim D I. Coordinated multipoint transmission in downlink multi-cell NOMA systems: models and spectral efficiency performance. IEEE Wirel Commun, 2018, 25: 24-31

54 Nguyen V D, Tuan H D, Duong T Q, et al. Precoder design for signal superposition in MIMO-NOMA multicell networks. IEEE J Sel Areas Commun, 2017, 35: 2681-2695

55 Ding Z G, Fan P Z, Poor H V. Impact of user pairing on $5 \mathrm{G}$ nonorthogonal multiple-access downlink transmissions. IEEE Trans Veh Technol, 2016, 65: 6010-6023

56 Ding Z G, Schober R, Poor H V. Unveiling the importance of SIC in NOMA systems: Part I — state of the art and recent findings. 2020. ArXiv:2005.10215

57 Salehi M, Tabassum H, Hossain E. Accuracy of distance-based ranking of users in the analysis of NOMA systems. IEEE Trans Commun, 2019, 67: 5069-5083

58 Guo S J, Zhou X W. Robust resource allocation with imperfect channel estimation in NOMA-based heterogeneous vehicular networks. IEEE Trans Commun, 2019, 67: 2321-2332

59 Azari M M, Rosas F, Chen K C, et al. Ultra reliable UAV communication using altitude and cooperation diversity. IEEE Trans Commun, 2018, 66: 330-344

60 Usman M R, Khan A, Usman M A, et al. On the performance of perfect and imperfect SIC in downlink non orthogonal multiple access (NOMA). In: Proceedings of International Conference on Smart Green Technology in Electrical and Information Systems (ICSGTEIS), Bali, 2016. 102-106

61 Mahady I A, Bedeer E, Ikki S, et al. Sum-rate maximization of NOMA systems under imperfect successive interference cancellation. IEEE Commun Lett, 2019, 23: 474-477

62 Chen X M, Jia R D, Ng D W K. On the design of massive non-orthogonal multiple access with imperfect successive interference cancellation. IEEE Trans Commun, 2019, 67: 2539-2551

63 Zhu J Y, Wang J H, Huang Y M, et al. On optimal power allocation for downlink non-orthogonal multiple access systems. IEEE J Sel Areas Commun, 2017, 35: 2744-2757

64 Yang Z, Ding Z G, Fan P Z, et al. A general power allocation scheme to guarantee quality of service in downlink and uplink NOMA systems. IEEE Trans Wirel Commun, 2016, 15: 7244-7257

65 Chen Z Y, Ding Z G, Dai X C, et al. An optimization perspective of the superiority of NOMA compared to conventional OMA. IEEE Trans Signal Process, 2017, 65: 5191-5202

66 Zamani M R, Eslami M, Khorramizadeh M, et al. Energy-efficient power allocation for NOMA with imperfect CSI. IEEE Trans Veh Technol, 2019, 68: 1009-1013

67 Fang F, Zhang H J, Cheng J L, et al. Joint user scheduling and power allocation optimization for energy-efficient NOMA systems with imperfect CSI. IEEE J Sel Areas Commun, 2017, 35: 2874-2885

68 Sun Y, Ng D W K, Ding Z G, et al. Optimal joint power and subcarrier allocation for MC-NOMA systems. In: Proceedings of IEEE Global Communications Conference (GLOBECOM), Washington, 2016

69 Salaün L, Chen C S, Coupechoux M. Optimal joint subcarrier and power allocation in NOMA is strongly NP-hard. In: Proceedings of IEEE International Conference on Communications (ICC), Kansas City, 2018

70 Liang W, Ding Z G, Li Y H, et al. User pairing for downlink non-orthogonal multiple access networks using matching algorithm. IEEE Trans Commun, 2017, 65: 5319-5332

71 Sedaghat M A, Muller R R. On user pairing in uplink NOMA. IEEE Trans Wirel Commun, 2018, 17: 3474-3486

72 Chen X, Gong F K, Li G, et al. User pairing and pair scheduling in massive MIMO-NOMA systems. IEEE Commun Lett, 2018, 22: 788-791

73 Bui V, Nguyen P X, Nguyen H V, et al. Optimal user pairing for achieving rate fairness in downlink NOMA networks. In: Proceedings of International Conference on Artificial Intelligence in Information and Communication (ICAIIC), Okinawa, 2019. $575-578$

74 Budhiraja I, Tyagi S, Tanwar S, et al. Cross layer NOMA interference mitigation for femtocell users in $5 \mathrm{G}$ environment. IEEE Trans Veh Technol, 2019, 68: 4721-4733

75 Shin W, Vaezi M, Lee B, et al. Non-orthogonal multiple access in multi-cell networks: theory, performance, and practical challenges. IEEE Commun Mag, 2017, 55: 176-183

76 Beylerian A, Ohtsuki T. Coordinated non-orthogonal multiple access (CO-NOMA). In: Proceedings of IEEE Global Communications Conference (GLOBECOM), Washington, 2016

77 Choi J. Non-orthogonal multiple access in downlink coordinated two-point systems. IEEE Commun Lett, 2014, 18: 313-316

78 Sun X F, Yang N, Yan S H, et al. Joint beamforming and power allocation in downlink NOMA multiuser MIMO networks. IEEE Trans Wirel Commun, 2018, 17: 5367-5381

79 Sun Y S, Ding Z G, Dai X C, et al. A feasibility study on network NOMA. IEEE Trans Commun, 2018, 66: 4303-4317

80 Ding Z G, Adachi F, Poor H V. The application of MIMO to non-orthogonal multiple access. IEEE Trans Wirel Commun, 2016, 15: $537-552$

81 Ding Z, Schober R, Poor H V. A general MIMO framework for NOMA downlink and uplink transmission based on signal alignment. IEEE Trans Wirel Commun, 2016, 15: 4438-4454

82 Ding Z, Poor H V. Design of massive-MIMO-NOMA with limited feedback. IEEE Signal Process Lett, 2016, 23: 629-633

83 Badrudeen A A, Leow C Y, Won S H. Performance analysis of hybrid beamforming precoders for multiuser millimeter wave NOMA systems. IEEE Trans Veh Technol, 2020, 69: 8739-8752

84 Khoshkholgh M G, Navaie K, Shin K G, et al. Coverage analysis of multi-stream MIMO hetnets with MRC receivers. IEEE Trans Wirel Commun, 2017, 16: 7816-7833

85 Gong M Y, Yang Z. The application of antenna diversity to NOMA with statistical channel state information. IEEE Trans Veh Technol, 2019, 68: 3755-3765

86 Cherif N, Alzenad M, Yanikomeroglu H, et al. Downlink coverage and rate analysis of an aerial user in integrated aerial and terrestrial networks. 2019. ArXiv:1905.11934 